\title{
Evidence for the release of gastric fibrinolytic activity into peripheral blood
}

\author{
H. T. COX, L. POLLER ${ }^{1}$, AND JEAN M. THOMSON \\ From Wythenshawe Hospital and the Haematology Department, Withington \\ Hospital, Manchester
}

Our previous paper (Cox, Poller, and Thomson, 1967) suggested that local gastric fibrinolysis may play a part in causing acute gastric bleeding without an obvious ulcer and perhaps also in causing chronic peptic ulceration. As a consequence of these results the need to investigate clinical cases of bleeding gastric ulcer for abnormal fibrinolysis has been suggested (Lancet, 1967).

It appeared preferable at this juncture, however, to study the problem of gastric fibrinolysis in more general terms because investigation of cases with gastric bleeding is subject to a number of difficulties: sudden blood loss by itself may produce shock and hence fibrinolytic activity; fibrinolytic enzymes are notoriously labile; and the time relationship to bleeding may be uncertain.

It was during consequent investigations of gastric fibrinolysis that we found that digital compression of the stomach at laparotomy produced markedly raised fibrinolytic activity in peripheral venous blood. In this paper we describe these findings and their possible clinical implications.

\section{METHOD OF STUDY}

Previously the gastric vein specimens were taken before any manipulation of the stomach. The stomach, however, is not a quiescent static organ. Pressures rise during peristalsis and vomiting. In the present study, before taking gastric vein specimens, we applied digital pressure to the stomach walls in an attempt to reproduce on them the effect of naturally occurring rises in pressure. In addition we carried out serial studies on peripheral venous blood to see whether the stomach can be a source of peripheral venous fibrinolytic activity.

The study was performed on 18 patients who underwent laparotomy. Twelve had chronic duodenal ulcers, two with associated pyloric obstruction; four patients had gallstones with no evidence of gastric or duodenal pathology; two patients were suspected of having a chronic duodenal ulcer because of radiological findings and clinical history, but no pathology in the duodenum, stomach, or gallbladder was seen at operation. The gallstones were removed by cholecystectomy; of the

\footnotetext{
${ }^{1}$ Requests for reprints should be sent to L.P.
}

12 patients with chronic duodenal ulcer, 10 were treated by vagotomy and pyloroplasty, two by vagotomy and gastrojejunostomy. The two patients with suspected duodenal ulcer were treated by vagotomy and pyloroplasty on the assumption that the diagnosis was recurrent acute ulceration.

The first step in the procedure was to pick up the stomach and to exert pressure on the anterior and posterior walls by compression between the finger and thumb, the finger anterior to the anterior wall and the thumb posterior to the posterior wall. It was not known whether gastric pressure would affect fibrinolytic activity, or if it did, for how long pressure should be exerted. An arbitrary decision was made to compress all areas of the stomach in turn by digital pressure from the pylorus to the fundus. This took 30 seconds. In 13 of the patients the stomach was compressed in this way and for this length of time before each gastric venous specimen was taken. In the last five cases compression was applied for no more than 10 seconds to the drainage areas of the selected veins only.

The gastric vein specimens were taken immediately after this gastric compression, all specimens being obtained within five minutes. The aim in each case was to take three specimens from each patient. In one obese patient no gastric vein specimen could be obtained; peripheral vein specimens were nevertheless taken. Usually two venous specimens were collected from the tributaries of the left gastric vein and one from the right gastroepiploic vein. Blood was taken from the latter after distal occlusion with finger and thumb. In all cases three specimens of peripheral venous blood were taken at 10-minute intervals and in five cases an additional fourth specimen was collected. The first peripheral venous specimen was taken at the same time as the first gastric vein specimen. All specimens were transferred to melting ice, kept at $0^{\circ} \mathrm{C}$, and tested within an hour.

\section{TECHNIQUE}

The laboratory methods were as before (Cox et al, 1967) using the standard fibrin plate, modified fibrin plate (incorporating epsilon amino-caproic acid), heated fibrin plate, and euglobulin lysis time techniques. Thrombelastograph studies on peripheral and gastric venous blood were also performed on eight of our patients. Gastric pressures were measured during compression by introducing an intragastric balloon attached to a U-tube water man404 
ometer, external pressure being applied to the gastric wall over the balloon. Care was taken to use a suitable balloon of such length and elasticity that the pressure on one part could not be associated with stretch in another part of the balloon, giving false low pressure recordings. The balloon was of sufficient capacity to ensure that it was not completely emptied of air at the pressures with which we were working, otherwise false low pressure recording would again have been possible.

Additional selective fibrin plates were used to differentiate fibrinolytic from possible peptic activity. These are not discussed in detail in this paper.

\section{RESULTS}

Fifty-nine serial specimens of peripheral venous blood and 44 of gastric venous blood were obtained from our patients.

GASTRIC VENOUS SPECIMENS At least one specimen of gastric vein blood from each patient showed increased gross fibrinolytic activity as measured by both the euglobulin lysis time and fibrin plate and free plasmin with the heated fibrin plate technique. The distribution of the results with the euglobulin lysis time in the serial gastric specimens is shown in Figure 1. This shows that the mean lysis times for each group of specimens was grossly shortened. The mean lysis time for gastric vein 1 was 33.3 minutes, for gastric vein 2 it was $\mathbf{4 4 . 8}$ minutes, and for gastric vein 3 it was 34.0 minutes. The shortest individual euglobulin lysis time was three minutes obtained in one of the two cases of peptic ulcer with pyloric stenosis. Of the total of 44 serial specimens, 38 gave positive zones of lysis on the standard plate and 37 on the heated plate.

Eight gastric venous specimens from five cases were tested on plates incorporating epsilon aminocaproic acid. All these specimens, which had shown positive zones on standard and heated plates, were inhibited by the antifibrinolytic drug, EACA.

The euglobulin lysis times were shorter and the zones of lysis greater than in our previous investigation of gastric vein blood when gastric compression was not applied.

PERIPHERAL VENOUS SPECIMENS A characteristic pattern emerged from the serial studies on peripheral blood. A progressive increase of activity was seen in euglobulin lysis times (Fig. 2) and on fibrin plates (Fig. 3) with specimens collected at 10-minute intervals. The highest activities were found in the third and fourth specimens, ie, 20 to 30 minutes after compression of the stomach.

The distribution of euglobulin lysis times is given in Figure 1. Figure 2 gives the mean results of lysis times of peripheral venous blood taken at four 10-minute intervals. It will be seen from this figure that the mean euglobulin lysis time of the first peripheral specimen was 158 minutes. The first peripheral specimen in one case only showed a zone of lysis on a standard fibrin plate, the heated plate being negative in this patient, ie, activator activity only was present. The second peripheral venous

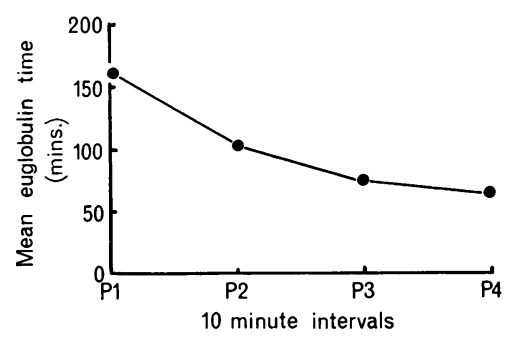

FIG. 2. Mean eugloblin lysis time of peripheral venous specimens.

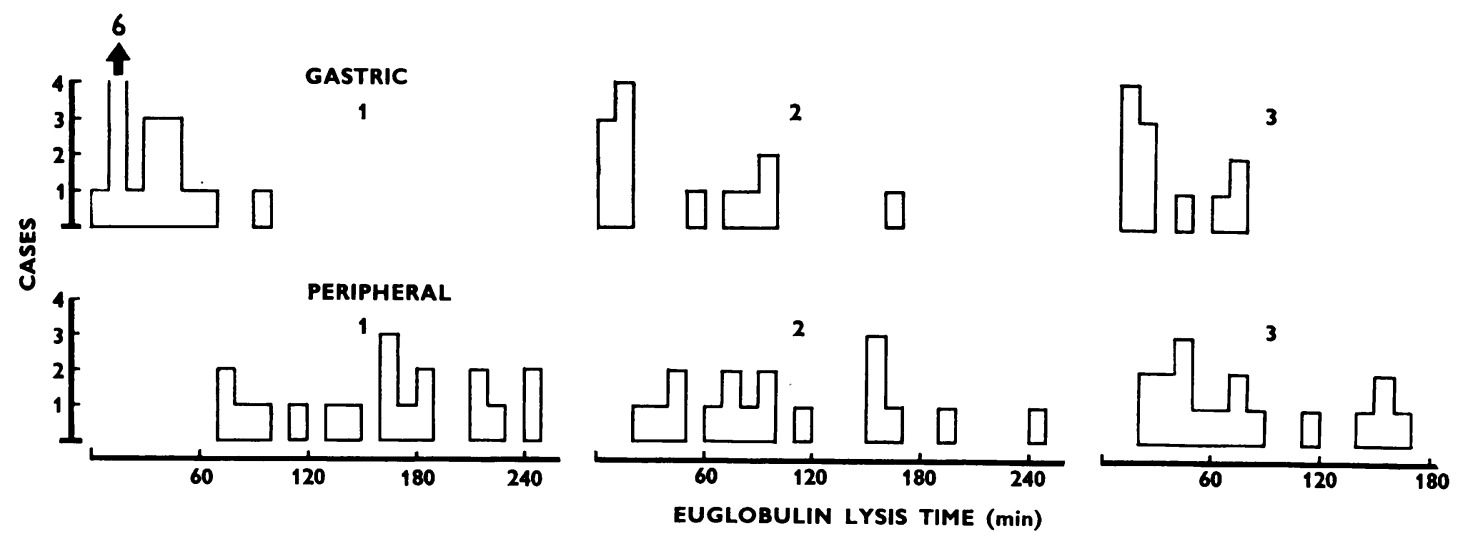

FIG. 1. Distribution of euglobulin lysis times of gastric and peripheral venous specimens. 


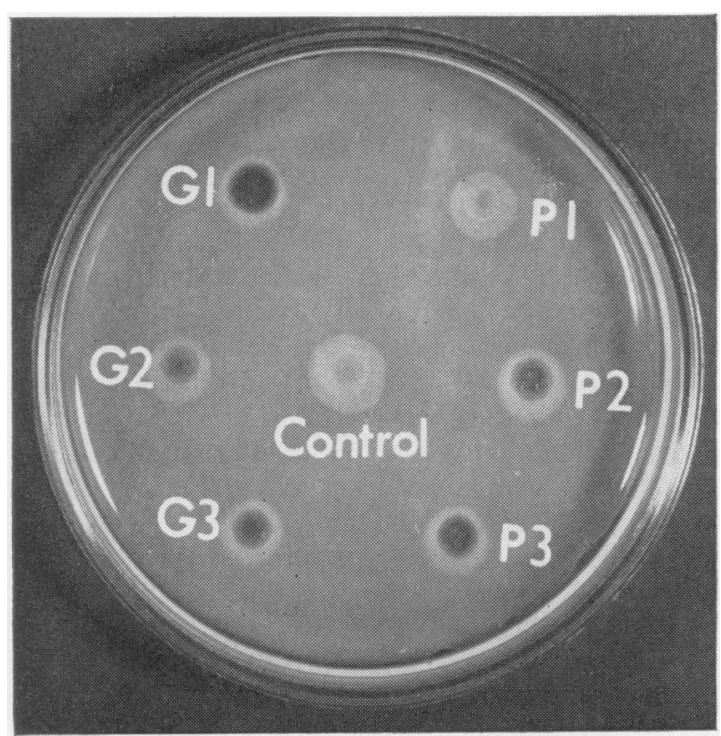

FIG. 3. Heated fibrin plate showing plasmin activity in gastric and peripheral venous blood.

PI

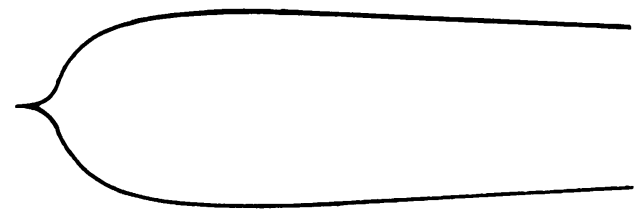

G I

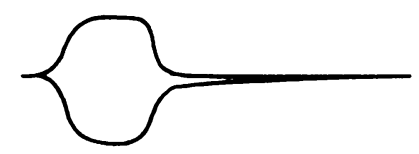

G2

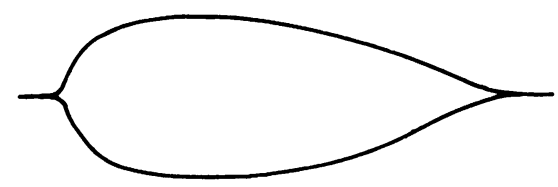

G3

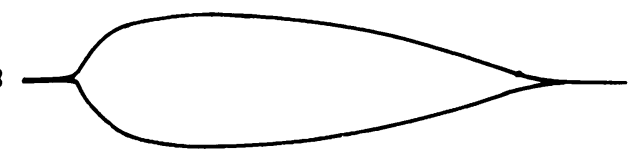

FIG. 4. Thromboelastograms of patient with grossly increased pattern of fibrinolysis in gastric vein specimens $(G 1, G 2, G 3)$. specimens, taken 10 minutes later, gave positive zones of lysis on a standard plate in seven of 18 patients and in five of these the heated plate was also positive, $i e$, plasmin was present. The third peripheral venous specimens taken after a further 10 minutes gave zones of lysis in nine of 17 cases on the standard plate, of which eight showed plasmin-like activity on the heated plate. Of the five specimens taken an hour after the first sample, three were positive on both standard and heated plates. In 10 of the 18 cases plasmin-like activity was found in at least one peripheral vein specimen. In the eight cases in which they were performed, thromboelastographic studies supported the findings on fibrin plates and results for euglobulin lysis time on both gastric and venous specimens. In one patient a pattern of grossly pathologically increased activity was obtained (Fig. 4). This was in one of the two patients with pyloric stenosis who had an abnormally short euglobulin lysis time $(8 \mathrm{~min})$ in the gastric vein blood.

GASTRIC PRESSURES The recordings of intragastric pressure at the time of gastric compression were between 80 and $100 \mathrm{~cm}$ of water. Pressure changes of a similar order, ie, 80 to $100 \mathrm{~cm}$ of water, were produced by retching or coughing, in a series of patients we recorded in a hospital ward. Elsewhere the normal range of intragastric pressure has been found to be between 10 and $60 \mathrm{~cm}$ water (Stunkard and Reinhard, 1965). A peak pressure of $200 \mathrm{~mm}$ of mercury ( $271.4 \mathrm{~cm}$ of water) is stated to occur during vomiting (Wells and Kyle, 1967).

\section{DISCUSSION}

The demonstration of the presence of fibrinolytic activity in peripheral venous blood and its progressive rise, following gastric compression, seems of considerable importance. The most significant finding here was the presence of plasmin-like activity in peripheral blood in the majority of our cases. This was not present in any of the first peripheral specimens but was present in nearly one third of the specimens taken after 10 minutes and in over a third of the 20- and 30-minute specimens. Figure 3 illustrates the gradation of this activity. Free plasmin is not usually present in peripheral blood except in gross defibrination states. Its presumed presence here is even more surprising than its presence in gastric vein blood which we reported previously. Evidence that this may be plasmin and not peptic or tryptic activity was obtained during this study and will be reported elsewhere.

The normal range of euglobulin lysis times in peripheral venous blood, using the same technique, lies between three and five hours. A euglobulin lysis 
time of $\mathbf{3 0}$ minutes is generally regarded as indicating excess fibrinolysis. Figure 1 shows that the lysis times in the third peripheral specimens were approximating to this level of activity.

The first peripheral blood was taken simultaneously with the first gastric sample, ie, immediately after compression, and showed a shortened lysis time. It is reasonable to assume that the lytic activity released from the stomach would not have reached the arm vein in sufficient concentration by this time, and that the shortened lysis times were due to the stress of operation. This view is supported by the fact that there was no significant difference in the first peripheral specimens taken in this study and the peripheral specimens in our previous study without compression of the stomach (Cox et al, 1967). It is also important to note that in no case was free plasmin detected in the first peripheral venous specimen.

Our earlier study showed that the lytic activity in gastric vein blood was raised in patients with peptic ulcer compared with a non-ulcer group. Compression of the stomach at operation has produced plasmin-like activity in at least one gastric vein specimen of every patient, including the non-ulcer cases. This indicates the fibrinolytic potential of the stomach in patients without gastric pathology. Even so, there appeared a gradation of fibrinolytic activity, the levels being highest in patients with peptic ulcer. This again suggests an aetiological relationship between raised fibrinolysis and peptic ulcer and that antifibrinolytic therapy may be of value in the medical management of peptic ulcer and acute gastric haemorrhage.

Over half the gastric vein specimens gave euglobulin lysis times of less than half an hour. Euglobulin lysis times of three and eight minutes, the shortest times we obtained, were in the gastric veins of the two cases of peptic ulcer with pyloric stenosis, where excessive motility is likely. These must be accepted as dramatic results. In the last five cases in our study, the duration of gastric compression was considerably less than in the first thirteen. There was no indication that the resulting lytic activity was less in these patients so it does not appear that the duration of gastric compression is critical.

Whatever the aetiological implications with regard to acute haematemesis and chronic ulceration the demonstration that the stomach is a source of fibrinolysis in peripheral blood may well have practical implications of a different nature. By digital compression of the stomach the abdominal surgeon may have at his disposal a simple method of raising peripheral venous fibrinolytic activity. This may diminish the incidence of postoperative thrombosis. The 30 -minute euglobulin lysis time is the conventional aim in thrombolytic therapy. It is interesting, therefore, that this level can be achieved by compression of the stomach. The duration of this rise of fibrinolytic activity is obviously of clinical importance. In no case in our series, however, was there an obvious defect in wound haemostasis after gastric compression.

\section{SUMMARY}

Studies have been performed on peripheral venous blood to determine whether the excess fibrinolytic activity we demonstrated previously in gastric veins can reach and be demonstrated in the peripheral circulation. Digital gastric compression has been performed at laparotomy and we have been able to demonstrate plasmin-like activity in the peripheral veins and a progressive shortening of the euglobulin lysis time. The stomach has not been previously regarded as a source of fibrinolytic activity.

Plasmin-like activity was found in at least one gastric vein specimen of every case but there was a gradation of activity, the higher levels being found in gastric ulcer cases. This finding again suggests a relationship between fibrinolytic activity and peptic ulcer and the possible value of antifibrinolytic drugs in the management of gastric bleeding.

Whatever the aetiological implications with regard to gastric bleeding and ulceration it does appear that the surgeon may have at his disposal a simple method of raising peripheral fibrinolytic activity which may reduce postoperative thrombosis.

This work was assisted by a grant for thrombosis research to one of us (L.P.) from the Manchester Regional Hospital Board. We also wish to thank T. Bhaduri, surgical registrar, and G. W. Bradley, house surgeon, of Wythenshawe Hospital for performing the gastric pressure readings.

\section{REFERENCES}

Cox, H.T., Poller, L., and Thomson, J. M. (1967). Gastric fibrinolysis. Lancet, 1, 1300-1302.

Stunkard, A. J., and Reinhard, A. (1965). Effects of an intragastric balloon on gastric pressures in man. J. appl. Physiol., 20, 1345-1350.

Wells, C., and Kyle, J. (1967). Scientific Foundations of Surgery, p. 163. Heinemann, London. 